\title{
Old drug, new data
}

\author{
REVISITING... LITHIUM THERAPY
}

I. Nicol Ferrier, Lindsey J. Ferrie \& Karine A. Macritchie

Abstract New data have emerged over the past 10 years regarding the efficacy and mechanisms of action of lithium. This article briefly summarises the evidence for the use of lithium to treat affective disorders and psychosis, reviews its putative anti-suicidal effect, highlights new research on its mechanism of action and provides an update on some important side-effects and consequences of its use.

The authors revisit a topic considered in the first volume of APT (Ferrier et al, 1995). That 1995 article is available on our website (http://apt. rcpsych.org) as a data supplement to the online version of the present submission.

Lithium remains a mainstay in the acute and prophylactic treatment of bipolar affective disorder. It is used in the augmentation of antidepressant treatment and, less frequently, in the augmentation of antipsychotic treatment of schizophrenia. It is reported to have specific anti-suicidal effects. Systematic reviews by the Cochrane Collaboration and others have examined the evidence base for its use in these contexts. A brief summary of their findings is given here.

\section{Evidence of efficacy}

\section{Maintenance treatment}

In a systematic review Burgess et al (2001) investigated the efficacy of lithium in preventing recurrence of mood disorders. They included nine randomised placebo-controlled studies of lithium involving a total of 825 participants. About half of the trials had recruited people with bipolar disorder, and the remainder studied people with unipolar disorder or mixed groups with either unipolar or bipolar disorder. Participants received treatment for at least 3 months and blood serum lithium concentrations ranged from 0.5 to $1.4 \mathrm{mmol} / 1$. Lithium had greater efficacy than placebo in relapse prevention for mood disorders in general, but the effect size was modest. The most consistent effect was found in bipolar disorder. In unipolar disorder, the trend favoured lithium, but its effect was not statistically significant.

\section{Treatment and prevention of mania}

In the treatment of mania, one randomised controlled trial (Bowden et al, 1994) found that a greater proportion of patients responded to lithium than to placebo. Geddes (2004) summarised the data from systematic reviews and from randomised controlled trials using lithium in comparison with other active agents. Lithium was superior to chlorpromazine but less effective than risperidone in reducing manic symptoms. The data did not demonstrate a significant difference in antimanic efficacy between lithium and haloperidol, carbamazepine or valproate.

Twoagents, lamotrigine and olanzapine, merit more detailed discussion, as new evidence has emerged. Three randomised controlled trials have examined lamotrigine's efficacy in mania. Although Ichim et al (2000) found no difference in antimanic effect between lithium and lamotrigine, two unpublished studies in mania found no significant difference between lamotrigine and placebo (GlaxoSmithKline Clinical Trial Register: http://ctr.gsk.co.uk/Summary/ lamotrigine/studylist.asp). Goodwin et al (2004)

Nicol Ferrier is Professor of Psychiatry and Head of the School of Neurology, Neurobiology and Psychiatry at the University of Newcastle upon Tyne (Leazes Wing, Royal Victoria Infirmary, Queen Victoria Road, Newcastle upon Tyne NE1 4LP, UK. Email: i.n.ferrier@ncl.ac.uk) and Honorary Consultant Psychiatrist for Newcastle, North Tyneside and Northumberland Mental Health NHS Trust. He works in a clinical capacity for a regional service for patients with chronic affective disorders. His research interests are in psychopharmacology and the neurobiology and treatment of severe affective disorders. He collaborated on the National Institute for Health and Clinical Excellence guidelines for unipolar depression and is Chairman of the team preparing similar guidelines for bipolar disorder. Lindsey J. Ferrie was awarded a PhD from the University of Newcastle upon Tyne for her thesis on the effect of chronic lithium treatment on the dopaminergic system. Karine A. Macritchie studied physiology and medicine at the University of Glasgow. She undertook basic training in psychiatry at Cambridge and recently completed specialist training in general adult psychiatry and liaison psychiatry at Newcastle upon Tyne. Her research interests include affective disorders, women's mental health, evidence-based medicine and neuroimaging. 
found that lithium had prophylactic efficacy against mania, prolonging the time to intervention in a pooled analysis of two placebo-controlled 18-month trials of lamotrigine and lithium in bipolar I disorder. Although both lithium and lamotrigine delayed the time to treatment for any mood episode, lamotrigine showed a more robust effect in preventing depression than mania.

In studies comparing lithium and olanzapine, one 4-week randomised controlled trial found no significant difference in their effects in the treatment of mania (Berk et al, 1999). A 12-month study comparing these agents in maintenance treatment of people recently recovered from mania revealed some benefit for olanzapine (Tohen et al, 2005): it was significantly more effective in preventing mania and mixed episode recurrence, but not in preventing depression. It was also associated with significantly more weight gain.

\section{Treatment of bipolar depression}

Although several expert guidelines recommend lithium for the treatment and prevention of depression in bipolar disorder, the evidence from randomised controlled trials for its use in acute bipolar depression is surprisingly limited. It is based on a small number of early placebo-controlled trials, none of which is of parallel-group design. A relatively recent systematic review on bipolar depression found no randomised controlled trials to be of methodological quality sufficient to provide reliable evidence of its efficacy (Nolen \& Bloemkolk, 2000). However, lack of evidence does not in itself establish lack of effectiveness and there is indirect evidence of lithium's efficacy in this context. For instance, in one study, people in the depressed phase of bipolar disorder with high serum lithium levels showed no significant difference in response rates from those treated with antidepressants (Nemeroff $e t$ $a l, 2001)$. Its prophylactic efficacy against depressive recurrence also suggests antidepressant activity (Burgess et al, 2001).

\section{Augmentation in treatment-resistant depression}

Bauer \& Döpfmer (1999) found evidence in support of lithium augmentation in the treatment of refractory depression. Their systematic review included nine randomised placebo-controlled trials, with a total of 234 patients. The duration of treatment ranged from 2 to 42 days. In three trials, a minimum daily dose of $800 \mathrm{mg}$ was used or a target serum lithium concentration of greater than $0.5 \mathrm{mEq} / 1$ was achieved for 2 weeks: lithium augmentation led to a significantly higher response rate than placebo. When all nine studies were entered into a cumulative meta-analysis in order of increasing dose, the effect of lithium treatment was statistically significant at a daily dose of $600-800 \mathrm{mg}$. Bauer \& Döpfmer concluded that lithium augmentation increased treatment response in people with refractory depression.

\section{Schizophrenia}

The efficacy of lithium in schizophrenia was analysed by Leucht et al (2004). They identified 20 randomised controlled trials, involving 611 participants, comparing lithium with antipsychotics or placebo. They found no evidence that lithium monotherapy is an effective treatment for schizophrenia. The evidence available on augmentation of antipsychotics with lithium was inconclusive.

\section{Anti-suicidal effects}

The anti-suicidal effects of lithium have been debated over the past 30 years. Tondo et al (2001) sought studies on lithium treatment in people with bipolar disorder, major affective disorder and schizoaffective disorder which reported data on suicide rates. They identified 22 studies, involving a total of 5647 participants with 33473 patient years. Only three of the studies were randomised. For comparison, they used data from 13 studies reporting suicide rates for patients who were not receiving lithium treatment over a mean period of 5 years. The suicide rate in the groups receiving lithium treatment was significantly lower than that in groups without lithium. Results consistent with a protective effect against suicide were also found when the analysis was restricted to the three randomised trials. A recent meta-analysis of 12 randomised studies reporting suicide rates in people with mood disorders provides new support for the protective effect of lithium in this context (Cipriani et al, 2005).

In a narrative review Muller-Oerlinghausen et al (2005) summarised the evidence for lithium's effects against suicide and suicidal behaviour in affective disorders, concentrating on bipolar disorder. The following interesting questions were raised: is the putative anti-suicidal effect of lithium specific to lithium and does it occur outwith improvements in affective symptoms?

In discussing whether the anti-suicidal effect of lithium is shared by other psychotropic agents, the authors cited two randomised controlled trials. First, a randomised prospective study compared lithium with carbamazepine in the treatment of bipolar and schizoaffective disorders over 21/2 years (Multicenter Study of Long-term Treatment of Affective and 
Schizoaffective Psychoses (MAP); Thies-Flechtner et al, 1996). There were significantly more suicides and suicide attempts in the carbamazepine group. Second, Goodwin et al (2003) conducted a retrospective review of suicide risk in patients on lithium compared with those on valproate and those on carbamazepine. The adjusted suicide risk for the valproate group was 2.7 times higher than that for the lithium group. The study was insufficiently powered to allow similar comparisons for carbamazepine.

Does lithium have an anti-suicidal effect independent of its effects in episode treatment and prevention? Ahrens \& Muller-Oerlinghausen (2001) conducted a sub-analysis of data from the International Group for the Study of Lithium-treated Patients. They selected individuals with recurrent affective disorders and at least one suicide attempt before the onset of lithium treatment (a total of 176) and divided them into three groups on the basis of their response to lithium (excellent, moderate or poor). A statistically significant reduction in suicide attempts was found in all three groups, even those who were considered to have responded poorly to treatment. Although clearly not conclusive, these data support an independent suicidal effect for lithium.

\section{Summary of the evidence}

Box 1 summarises the evidence base from systematic reviews of lithium in affective disorders, psychosis and against suicide.

\section{Mechanism of action: progress on understanding}

Despite lithium's established therapeutic efficacy, details of its therapeutic mechanism of action are yet to be determined. Lithium has been shown to produce a number of biochemical effects, including effects on monoamines, other neurotransmitters, second messengers and regulators of intracellular signalling (Table 1). However, which, or which combinations of, effects contribute to its therapeutic response(s) remains unclear.

\section{Monoamine neurotransmitter systems}

Because of the implication of monoamine neurotransmitter systems in the aetiology of the major psychiatric disorders, the interaction between lithium and monoamine neurotransmission has been the focus of much attention. Studies have revealed that lithium has the ability to modulate a number of components of central monoamine neurotransmission. However, much of this work is

\section{Box 1 Summary of the evidence}

Current systematic reviews of lithium studies suggest the following:

Affective disorders

- Lithium is an efficacious maintenance treatment for bipolar disorder, but the evidence for its prophylactic efficacy in unipolar disorder is less strong

- It is efficacious in the treatment of mania

- Its use in bipolar depression requires more methodologically rigorous examination

- Lithium augmentation increases treatment response in refractory depression

Psychoses

- Lithium monotherapy is not an effective treatment for schizophrenia

- The evidence on its use in the augmentation of antipsychotics is inconclusive

Anti-suicide effect

- Lithium has a protective effect against suicide and suicidality that may be directly pharmacological, rather than the consequence of symptom improvement or dependent on prolonged use in treatmentadherent patients

old and has inconsistencies, with different studies producing contradictory results, suggesting a need for more work to be carried out.

Preclinical and clinical studies suggest that, in the short term, lithium can increase serotonergic neurotransmission (Price et al, 1990; Shiah \& Yatham, 2000). However, this effect is not always evident after long-term treatment and therefore the implications of this in the mechanism of action of lithium in longterm treatment and in its anti-suicide effect are unclear (Lenox \& Manji, 1995). Furthermore, some studies have indicated a decrease in serotonergic neurotransmission after lithium treatment. These conflicting results are probably due to major differences in methodologies (administration, brain region, dose regimens, etc.).

Lithium can also affect the dopaminergic system. Acute administration of lithium has been shown to produce a decrease in dopamine neurotransmission that may link to its antimanic effect (Berggren, 1985). However, chronic administration of lithium has little consistent effect on dopamine turnover (Ferrie et al, 2005). Chronic lithium treatment does prevent behavioural and biochemical manifestations of haloperidol-induced dopamine receptor supersensitivity (Lenox \& Ha hn, 2000). It appears that 


\begin{tabular}{|c|c|c|}
\hline System & Effect of lithium & Reference \\
\hline 5-HT (serotonin) function & Greatly increased & Price et al, 1990; Shiah \& Yatham, 2000 \\
\hline Acetylcholinesterase function & Greatly increased & Dziedzicka-Wasylewska et al, 1996; Kameda et al, 2001 \\
\hline Sodium function & Increased & Wood \& Goodwin, 1987 \\
\hline Dopamine function & Reduced & Dziedzicka-Wasylewska et al, 1996; Kameda et al, 2001 \\
\hline GABA function & Increased & Motohashi, 1992; Antonelli et al, 2000 \\
\hline Inositol & Reduced & Hallcher \& Sherman, 1980 \\
\hline cAMP & Reduced & Goldberg et al, 1988; Chen et al, 2000 \\
\hline Protein kinase $\mathrm{C}$ & Reduced & Manji \& Lenox, 1999; Wang et al, 2001 \\
\hline Glycogen synthase kinase & Greatly reduced & Ryves \& Harwood, 2001 \\
\hline Brain-derived neurotrophic factor & Increased & Mai et al, 2002; Chuang, 2004 \\
\hline $\mathrm{Bcl}-2$ & Increased & Manji \& Chen, 2002 \\
\hline Pro-aptotic proteins (p53, BAX) & Reduced & Chuang et al, 2002 \\
\hline
\end{tabular}

this effect is mediated presynaptically, since lithium treatment has not been shown to result in any consistent change in $\mathrm{D}_{1}$ or $\mathrm{D}_{2}$ receptor regulation, which would have suggested a postreceptor site of lithium action (Dziedzicka-Wasylewska et al, 1996).

Clinical evidence suggests that the neurotransmission imbalance implicated in affective illness is related to excessive dopaminergic activity but also to a reduced cholinergic activity (Bymaster \& Felder, 2002). Chronic lithium administration produces an upregulation of muscarinic receptors in the rat brain (De Bruin et al, 2000). Studies have also shown that lithium treatment enhances various behavioural responses that are cholinergically mediated. Thus, lithium reduces the convulsant threshold to the nonspecific cholinergic muscarinic agonist arecoline and to anticholinesterases (Jope, 1993). There are also reports (Wang et al, 1997) that show that the sensitivity of central nicotinic receptors is decreased following lithium administration.

Although relatively less attention has been paid to the effects of lithium on amino acid and neuropeptide regulation in the brain, investigators have reported that lithium produces elevations in $\gamma$-aminobutyric acid (GABA) in the striatum and midbrain (Ahluwalia \& Singhal, 1981). An increase in GABA turnover in the hippocampus and striatum following lithium treatment has also been reported (Motohashi, 1992). This may be clinically important in bipolar disorder, given GABA's inhibitory actions.

\section{Effect on second messengers/intracellular signalling}

In recent years, it has become increasingly appreciated that modulation of neurotransmitter function and neuronal activity by intracellular signalling systems is a possible therapeutic action of lithium. It has long been established that lithium inhibits the intracellular enzymes inositol monophosphatase, cyclic adenosine monophosphate (cAMP) and protein kinase $C$, and consequently has numerous effects on neuronal activity that may link to its unique clinical profile (Gurvich \& Klein, 2002).

More recently, lithium has been shown to be an inhibitor of glycogen synthase kinase-3 $\beta$ (GSK$3 \beta$ ). This enzyme is an important mediator of a number of cellular processes, including regulation of transcription factors associated with apoptosis and neuronal plasticity. This may be the mechanism whereby lithium protects rat brain neurons in culture from glutamate-induced $\mathrm{N}$-methyl-D-aspartate (NMDA) receptor-mediated excitotoxicity. This neuroprotection is accompanied by upregulation of the anti-apoptopic factor Bcl-2 and activation of other cell survival factors. Lithium also induces the expression of brain-derived neurotrophic factor (BDNF) and subsequent activation of Trk$\mathrm{B}$, the BDNF receptor. The activation of BDNF/ Trk-B signalling is likely to be the mechanism of the neuroprotective effects of lithium that have been shown in rodent models of disease such as the rat model of stroke (Chuang, 2004). Whether these effects are seen in patients on lithium is not yet clear and the clinical significance of changes in cell signalling remains to be determined. However, there is preliminary evidence that lithium induces increases in grey-matter volume after 4 weeks in people with bipolar disorder (Moore et al, 2000). These changes may be particularly significant given the increasing evidence of structural changes in the brain and associated neuropsychological deficits even in euthymic bipolar disorder (Thompson et al, 2005). 
It is now accepted that lithium can regulate multiple targets. Some of these have been described above, but we are still some away from delineating just how the different actions of lithium combine to achieve its mood-stabilising effects.

\section{Adverse effects}

Lithium has a narrow therapeutic index and a number of adverse effects that often result in poor adherence to treatment (McCreadie et al, 1985). Table 2 outlines the more common or important non-toxic adverse effects, with their usual time of onset and relationship to serum levels. In the initial phase of treatment, thirst, abdominal discomfort, nausea, diarrhoea, gustatory disturbance, muscle weakness and fatigue may be troublesome. These symptoms occur more frequently at higher serum lithium levels. They tend to be transitory and are to be distinguished from symptoms of toxicity and from problematic longer-term effects such as tremor, diabetes insipidus, renal impairment and hypothyroidism.

Since the publication of our original review (Ferrier et al, 1995) new information on adverse effects has become available. We give below a brief review of our current knowledge and discuss new findings on lithium toxicity and renal impairment. We also review the clinical implications of the lithium 'rebound' phenomenon, an important adverse effect.

\section{Lithium toxicity}

Lithium toxicity (lithium intoxication) varies in presentation. Acute toxicity can occur in lithiumnaïve individuals or after a long, uneventful course of treatment. It can occur at therapeutic serum levels, and it can arise following a change in dose or the introduction of new medication. Chronic toxicity can be insidious but its effects are potentially serious.

Mild toxicity is characterised by nausea, diarrhoea, blurred vision, polyuria, dizziness, a fine resting tremor, muscle weakness or drowsiness. Cerebellar signs are a core feature of lithium neurotoxicity. With increasing severity, parkinsonism and chorea may occur, indicating basal ganglia involvement. Confusion, blackouts, fasciculation, hyper- reflexia, incontinence and hypernatraemia may develop. In severe cases, acute renal failure, hypotension or hypertension occur. Electrocardiogram changes include sinus and junctional bradycardia and heart block. Impaired consciousness, seizures and coma may progress to death. Neurotoxic effects may be insidious in onset. A persistent cerebellar ataxia has been reported in the absence of overt toxicity (Lang \& Davis, 2002).

\section{Risk factors}

Risk factors for lithium toxicity include factors that in themselves lead to increments in lithium serum levels and those that predispose to lithium toxicity at a given level. Nephrogenic diabetes insipidus and impaired creatinine clearance may result in increased lithium levels at a given dose. Similarly, drug interactions predisposing to toxicity include medications that cause hyponatraemia and reduced lithium clearance, for example frusemide, thiazide diuretics and some antidepressants. Angiotensin-converting enzyme (ACE) inhibitors reduce glomerular perfusion pressure and may enhance the tubular reabsorption of lithium. An

\section{Table 2 Early-, medium-term and late-onset side-effects of lithium, not associated with toxicity}

\begin{tabular}{|c|c|c|}
\hline Early (1-14 days) & Medium term (14-365 days) & Late ( $>365$ days) \\
\hline \multicolumn{3}{|c|}{ Related to high lithium level $(\geqslant 0.8 \mathrm{mmol} / \mathrm{l})$} \\
\hline \multirow[t]{5}{*}{$\begin{array}{l}\text { Gastrointestinal: anorexia; } \\
\text { bad taste in mouth; diarrhoea }\end{array}$} & Gastointestinal: vomiting; dry mouth & $\begin{array}{l}\text { Cognitive: slow reaction times; } \\
\text { impaired memory }\end{array}$ \\
\hline & Renal: polyuria; nephrogenic diabetes insipidus & Renal: impairment \\
\hline & Neurological: tremor & $\begin{array}{l}\text { Neurological: myopathy; } \\
\text { ataxia }\end{array}$ \\
\hline & Cardiac: T-wave inversion; dysrhythmia & \\
\hline & $\begin{array}{l}\text { Dermatological: worsening of skin diseases, } \\
\text { e.g. psoriasis }\end{array}$ & \\
\hline \multicolumn{3}{|l|}{ Not related to high lithium level } \\
\hline \multirow[t]{4}{*}{ Thyroid: reduced $\mathrm{T}_{4}$ (transient) } & $\begin{array}{l}\text { Thyroid: non-toxic goitre; hypothyroidism; } \\
\text { hyperthyroidism (rare) }\end{array}$ & Metabolic: weight increase \\
\hline & Gastrointestinal: persistent mild diarrhoea & \\
\hline & Haematological: leucocytosis & \\
\hline & Neurological: weakness & \\
\hline
\end{tabular}


important, potentially toxic, interaction is between lithium and non-steroidal anti-inflammatory drugs. Factors predisposing to lithium toxicity at a given dose include pre-existing brain injury and physical illnesses such as hypertension, diabetes and congestive cardiac failure.

\section{Diagnosis, treatment and sequelae}

Patients with suspected lithium toxicity should be observed for at least $24 \mathrm{~h}$. Lithium should be stopped, or the dose reduced. Lithium levels should be monitored immediately and then every $6-12 \mathrm{~h}$, depending on the presentation. The diagnosis of lithium toxicity is made on clinical grounds. A therapeutic level does not exclude toxicity. Serum lithium levels should not be used as the only guide to the severity of poisoning, as lithium absorption from the gastrointestinal tract and release from intracellular stores may be prolonged. Lithium excretion is greatly diminished in cases of toxicity, further compounding the problem.

In mild cases, attention to hydration, electrolyte balance and electrocardiogram (ECG) monitoring is necessary. In more severe cases, haemodialysis should be considered: it should be instituted when serum lithium levels are greater than $3 \mathrm{mmol} / 1$, in cases of coma or shock, or when conservative measures have failed. It is essential to observe symptom progression over time. Clinical improvement may take up to 3 weeks. Neurological symptoms occasionally persist, usually in the form of cerebellar symptoms, with the possibility of improvement over the following year. Persistence of neurological symptoms is more likely in more severe and prolonged cases of acute toxicity. The most commonly reported irreversible sequel of lithium neurotoxicity is persistent cerebellar dysfunction (Adityanjee et al, 2005).

\section{Renal impairment}

Lithium therapy may result in renal impairment through its effects on tubular or glomerular function. These will be considered in turn.

\section{Tubular dysfunction}

Diabetes insipidus is characterised by polyuria and polydipsia. It results from renal tubular dysfunction or from inadequate pituitary production of antidiuretic hormone. Lithium-induced diabetes insipidus is predominantly nephrogenic in origin (Bendz \& Aurell 1999), although case reports of the neurogenic form exist (Posner \& Mokrzycki, 1996). The kidney loses its ability to concentrate urine. Risk factors include a long duration of treatment and polypharmacy. It may occur less frequently with once-daily dosing (Bowen et al, 1991). Diagnosis is confirmed through the water deprivation test and the desmopressin (DDAVP) test.

In mild, reversible cases, simple lithium dose reduction or once-daily dosing may beadequate. Alternative strategies include combination psychotropic treatment or lithium substitution. In severe cases of acute diabetes insipidus, marked dehydration may occur (Bendz \& Aurell, 1999), necessitating referral to a renal physician. The priority is the restoration of water and electrolyte balance. In severe cases, the potential for lithium toxicity (exacerbated by dehydration), neurological impairment and encephalopathy should be recognised. Thiazide diuretics, although effective, should be avoided if possible, because an associated increased sodium and lithium reabsorption may lead to lithium toxicity.

Patients on long-term treatment may suffer a progressive urinary-concentrating impairment which is not fully reversible on stopping treatment. This impairment is thought to be due to irreversible underlying renal damage, and not to nephrogenic diabetes insipidus. There are reports of a correlation between impaired concentrating ability and the duration of lithium treatment (Hestbech et al, 1977). Hansen et al (1979) suggested an association between this functional impairment and chronic interstitial nephropathy.

Chronic interstitial nephropathy is frequently reported in lithium-associated renal disease. Its histopathological features include tubular atrophy and interstitial fibrosis. Although these features have been found in psychiatric patients who have never received lithium, distal tubular dilatation and microcyst formation appear to differentiate the lithium-treated group. Markowitz et al (2000) demonstrated that lithium-associated chronic tubulo-interstitial nephropathy may lead to renal insufficiency and renal failure, despite the discontinuation of treatment.

\section{Glomerular dysfunction}

The incidence of lithium-induced glomerular impairment is unclear owing to methodological inconsistencies between studies. The literature on lithium-induced glomerulosclerosis has been largely confined to case reports (Santella et al, 1988). However, a newer study on 24 patients with lithium nephrotoxicity found focal segmental glomerulosclerosis correlating with proteinuria (of $>1 \mathrm{~g} / \mathrm{dl}$ ) in half of the sample (Markowitz et al, 2000). These people had been treated with lithium for a mean of 13.6 years (range $2-25$ years). They all presented with renal insufficiency. Only two were known to have had previous episodes of acute lithium intoxication. These findings require confirmation in other studies, but it is possible that the incidence of lithium-induced glomerular toxicity has been underestimated. 


\section{Clinical implications}

Clinicians should remain alert to the possibility of renal failure in patients on lithium treatment. Acute renal failure associated with lithium toxicity may present with acute oliguria, fluid retention, uraemia and associated nausea and vomiting. Chronic renal failure may be asymptomatic in the early phases, with insidious onset of hypertension, thirst, polyuria and proteinuria. Risk factors for renal impairment include long duration of therapy, concomitant use of other medications, episodes of lithium toxicity and other coexisting medical conditions.

In patients with evidence of renal impairment, lithium levels should be monitored regularly (at least every 2 months) and kept at the lower end of the therapeutic range. Once-daily dosing is recommended. Measurement of $24 \mathrm{~h}$ urinary volume and creatinine clearance should be considered annually. In acute renal failure, an urgent nephrology opinion is indicated. Lithium should be stopped and an alternative treatment instituted, with appropriate dose adjustment for the degree of renal failure. Close collaboration with renal physicians may be required in the case of those in whom the benefits of treatment must be weighed against compromised renal function.

\section{‘Rebound' affective episodes on lithium discontinuation}

Abrupt discontinuation of lithium prophylaxis may precipitate early recurrence of mania and depressive episodes (Mander \& Loudon, 1988; Suppes et al, 1991). This occurrence, called the rebound phenomenon, presents a significant clinical problem. Goodwin (1994) argued that it may negate the benefits of lithium treatment altogether in bipolar disorder. Using data from Mander \& Loudon (1988) he calculated that, given the risk of precipitating early recurrence on its withdrawal compared with the risk of recurrence in the untreated group, lithium prophylaxis must be maintained for at least 24 months before an overall advantage is conferred. It appears that there is an earlier and greater risk of mania than of depression on lithium withdrawal (Mander \& Loudon, 1988; Suppes et al, 1991). Discontinuation of lithium may result in resistance to subsequent treatment with the drug, although this possibility is disputed (Coryell et $a l, 1998)$. There is also evidence that abrupt reduction of the dose of lithium should be avoided, as the relapse rate increases under this circumstance (Perlis et al, 2002).

Patients should be advised that abrupt discontinuation of lithium carries a high risk of manic recurrence. Gradual discontinuation over 4 weeks may lead to a lower recurrence rate (Faedda et al, 1993).

\section{Conclusions}

Lithium remains one of the most important weapons in our psychopharmacological armamentarium. Recent research has confirmed its efficacy in mania and in prophylaxis in bipolar disorder, and its useful role in treatment-resistant depression. There are also new insights into potential clinical benefits as a neuroprotective agent and as a way of reducing suicidal behaviour and suicide itself.

However, there is also an increasing understanding of the real and potential hazards of lithium therapy, including insidious and irreversible neuro- and nephrotoxicity. It is difficult for many patients to take the drug and, unfortunately, stopping it is not a neutral act - there are potential consequences so that the patient can be worse off than if they had never received lithium in the first place. Perhaps the way out of this dilemma - an effective evidence-based treatment but with significant negative consequences - lies in preclinical research, some of which is reviewed here. This work holds out the prospect of understanding lithium's mechanism(s) of action with the hope of thereby boosting its positive effects and diminishing the negative aspects, and/or the development of new, more effective but less toxic drugs.

\section{Declaration of interest}

None.

\section{References}

Adityanjee, Munshi, K. R. \& Thampy, A. (2005) The syndrome of irreversible lithium-effectuated neurotoxicity. Clinical Neuropsychopharmacology, 28, 38-49.

Ahluwalia, P. \& Singhal, R. L. (1981) Monoamine uptake into synaptosomes from various regions of rat brain following lithium administration and withdrawal. Neuropsychopharmacology, 20, 483-487.

Ahrens, B. \& Muller-Oerlinghausen, B. (2001) Does lithium exert an independent antisuicidal effect? Pharmacopsychiatry, 34, 132-136.

Antonelli, T., Ferioli, V., Lo Gallo, G., et al (2000) Differential effects of acute and short-term lithium administration on dialysate glutamate and GABA levels in the frontal cortex of the conscious rat. Synapse, 38, 355-362.

Bauer, M. \& Döpfmer, S. (1999) Lithium augmentation in treatment-resistant depression: a meta-analysis of placebocontrolled studies. Journal of Clinical Psychopharmacology, 19, 427-434.

Bendz, A. \& Aurell, M. (1999) Drug-induced diabetes insipidus: incidence, prevention and management. Drug Safety, 21, 449-456.

Berggren, U. (1985) Effects of chronic lithium treatment on brain monoamine metabolism and amphetamine induced locomotor stimulation in rats. Journal of Neural Transmission, 64, 239-250.

Berk, M., Ichim, M. \& Brook, S. (1999) Olanzapine compared to lithium in mania: a double-blind randomized controlled trial. Internal Clinical Psychopharmacology, 14, 339-343.

Bowden, C. L., Brugger, A. M., Swann, A. C., et al (1994) Efficacy of Divalproex vs lithium and placebo in the treatment of mania. $J A M A, 271,918-924$. 
Bowen, R. C., Grof, P. \& Grof, E. (1991) Less frequent lithium administration and lower urine volume. American Journal of Psychiatry, 148, 189-192.

Burgess, S., Geddes, J., Hawton, K., et al (2001) Lithium for maintenance treatment of mood disorders. Cochrane Database of Systematic Reviews, issue 3. Oxford: Update Software. DOI: 10.1002/14651858.CD003013.

Bymaster, F. P. \& Felder, C. C. (2002) Role of cholinergic muscarinic system in bipolar disorder and related mechanism of action of antipsychotic agents. Molecular Psychiatry, 7 (suppl. 1), S57-S63.

Chen, G., Masana, M. I. \& Manji, H. K. (2000) Lithium regulates PKC-mediated intracellular cross-talk and gene expression in the CNS in vivo. Bipolar Disorder, 2, 217-236.

Chuang, D. M. (2004) Neuroprotective and neurotrophic actions of the mood stabilizer lithium: can it be used to treat neurodegenerative diseases? Critical Reviews in Neurobiology, 16, 83-90.

Chuang, D. M., Chen, R. W., Chalecka-Franaszek, E., et al (2002) Neuroprotective effects of lithium in cultured cells and animal models of diseases. Bipolar Disorder, 4, 129-136.

Cipriani, A., Wilder, H., Hawton, K., et al (2005) Lithium in the prevention of suicidal behaviour and all-cause mortality in patients with mood disorders: a systematic review of randomised trials. American Journal of Psychiatry, 162, 18051819

Coryell, W., Solomon, D., Leon, A. C., et al (1998) Lithium discontinuation and subsequent effectiveness. American Journal of Psychiatry, 155, 895-898.

De Bruin, V. M. S., Marinho, M. M. F., De Sousa, F. C. F., et al (2000) Behavioural and neurochemical alterations after lithium-pilocarpine administration in young and adult rats. A comparative study. Pharmacology Biochemistry and Behavior 65, 547-551.

Dziedzicka-Wasylewska, M., Mackowiak, M., Fijar, K., et al (1996) Adaptive changes in the rat dopaminergic transmission following repeated lithium administration. Journal of Neural Transmission, 103, 765-776.

Faedda, G. L., Tondo, L., Baldessarinini, R. J., et al (1993) Outcome after rapid vs gradual discontinuation of lithium treatment in bipolar disorders. Archives of General Psychiatry, 50, 448-455.

Ferrie, L., Young, A. H. \& McQuade, R. (2005) Effect of chronic lithium and withdrawal from chronic lithium on presynaptic dopamine function in the rat. Journal of Psychopharmacology, 19, 229-234.

Ferrier, I. N., Tyrer, S. P. \& Bell, A. J. (1995) Lithium therapy. Advances in Psychiatric Treatment, 1, 102-108.

Geddes, J. R. (2004) Bipolar disorder. American Family Physician: Clinical Evidence Concise, 69, 1721-1722. http://www.aafp. org/afp/20040401/bmjx.html

Goldberg, H., Clayman, P. \& Skorecki, K. (1988) Mechanism of $\mathrm{Li}$ inhibition of vasopressin-sensitive adenylate cyclase in cultured renal epithelial cells. American Journal of Physiology, 255, F995-1002.

Goodwin, F. K., Fireman, B., Simon, G. E., et al (2003) Suicide risk in bipolar disorder during treatment with lithium and divalproex. JAMA, 290, 1467-1473.

Goodwin, G. (1994) Recurrence of mania after lithium withdrawal. Implications for the use of lithium in the treatment of bipolar affective disorder. British Journal of Psychiatry, 164, 149-152.

Goodwin, G. M., Bowden, C. L., Calabrese, J. R., et al (2004) A pooled analysis of 2 placebo-controlled 18-month trials of lamotrigine and lithium maintenance in bipolar I disorder Journal of Clinical Psychiatry, 65, 432-441.

Gurvich, N. \& Klein, P. S. (2002) Lithium and valproic acid: parallels and contrasts in diverse signalling contexts Pharmacology and Therapeutics, 96, 45-66.

Hallcher, L. M. \& Sherman, W. R. (1980) The effects of lithium ion and other agents on the activity of myo-inositol-1-phosphatase from bovine brain. Journal of Biological Chemistry, 255, 1089610901

Hansen, H. E., Hesbech, J., Sorensen, J. L., et al (1979) Chronic interstitial nephropathy in patients on long-term lithium treatment. Quarterly Journal of Medicine, 48, 577-591.

Hestbech, J., Hansen, H. E., Amdisen, A., et al (1977) Chronic renal lesions following long-term treatment with lithium. Kidney International, 12, 205-213.
Ichim, L., Berk, M. \& Brook, S. (2000) Lamotrigine compared with lithium in mania: a double blind randomized controlled trial. Annals of Clinical Psychiatry, 12, 5-10.

Jope, R. S. (1993) Lithium selectively potentiates cholinergic activity in rat brain. Progress in Brain Research, 98, 317-322.

Kameda, K., Miura, J., Suzuki, K., et al (2001) Effects of lithium on dopamine D2 receptor expression in the rat brain striatum. Journal of Neural Transmission, 108, 321-334.

Lang, E. J. \& Davis, S. M. (2002) Lithium neurotoxicity: the development of irreversible neurological impairment despite standard monitoring of serum lithium levels. Journal of Clinical Neuroscience, 9, 308-309.

Lenox, R. H. \& Hahn, C. G. (2000) Overview of the mechanism of action of lithium in the brain. Fifty-year update. Journal of Clinical Psychiatry, 61 (suppl. 9), 5-15.

Lenox, R. H. \& Manji, H. K. (1995) Lithium. In American Psychiatric Association Textbook of Psychopharmacology (eds A. F. Schatzberg \& C. B. Nemeroff), pp. 303-349. Washington, DC: American Psychiatric Press.

Leucht, S., McGrath, J. \& Kissling, W. (2004) Lithium for schizophrenia. Cochrane Library, issue 4. Oxford: Oxford Update Software.

Mai, L., Jope, R. S. \& Li, X. (2002) BDNF-mediated signal transduction is modulated by GSK3beta and mood stabilizing agents. Journal of Neurochemistry, 82, 75-83.

Mander, A. J. \& Loudon, J. B. (1988) Rapid recurrence of mania following abrupt discontinuation of lithium. Lancet, 2, 15-17.

Manji, H. K. \& Chen, G. (2002) PKC, MAP kinases and the bcl-2 family of proteins as long-term targets for mood stabilisers. Molecular Psychiatry, 7 (suppl. 1), S46-S56.

Manji, H. K. \& Lenox, R. H. (1999) Ziskind-Somerfeld Research Award. Protein kinase $C$ signalling in the brain: molecular transduction of mood stabilization in the treatment of manicdepressive illness. Biological Psychiatry, 46, 1328-1351.

Markowitz, G. S., Radhakrishnan, J., Kambham, N., et al (2000) Lithium nephrotoxicity: a progressive combined glomerular and tubulointerstitial nephropathy. Journal of the American Society of Nephrology, 11, 1439-1448.

McCreadie, R. G., McCormick, M. \& Morrison, D. P. (1985) The impact of lithium in South-West Scotland. III. The discontinuation of lithium. British Journal of Psychiatry, 146, 77-80.

Moore, G. J., Bebchuk, J. M., Hasanat, K., et al (2000) Lithium increases $\mathrm{N}$-acetyl aspartate in the human brain: in vivo evidence in support of bcl-2's neurotrophic effects? Biological Psychiatry, 48, 1-8.

Motohashi, N. (1992) GABA receptor alterations after chronic lithium administration. Comparison with carbamazepine and sodium valproate. Progress in Neuropsychopharmacology and Biological Psychiatry, 16, 571-579.

Muller-Oerlinghausen, B., Felber W., Berghofer, A., et al (2005) The impact of lithium long-term medication on suicidal behaviour and mortality of bipolar patients. Archives of Suicide Research, 9, 307-319.

Nemeroff, C. B., Evans, D. L., Gyulai, L., et al (2001) Double-blind, placebo-controlled comparison of imipramine and paroxetine in the treatment of bipolar depression. American Journal of Psychiatry, 158, 906-912.

Nolen, W. A. \& Bloemkolk D (2000) Treatment of bipolar depression: a review of the literature and a suggestion for an algorithm. Neuropsychobiology, 42 (suppl. 1), 11-17.

Perlis, R. H., Sachs, G. S., Lafer, B., et al (2002) Effect of abrupt change from standard to low serum levels of lithium: a reanalysis of double-blind lithium maintenance data. American Journal of Psychiatry, 159, 1155-1159.

Posner, L. \& Mokrzycki, M. H. (1996) Transient central diabetes insipidus in the setting of underlying chronic nephrogenic diabetes insipidus associated with lithium use. American Journal of Nephrology, 16, 339-343.

Price, L. H., Charney, D. S., Delgado, P. L., et al (1990) Lithium and serotonin function: implications for the serotonin hypothesis of depression. Psychopharmacology, 100, 3-12.

Ryves, W. J. \& Harwood, A. J. (2001) Lithium inhibits glycogen synthase kinase-3 by competition for magnesium. Biochemical and Biophysical Research Communications, 280, 720-725.

Santella, R. N., Rimmer, J. M. \& MacPherson, B. R. (1988) Focal segmental glomerulosclerosis in patients receiving lithium carbonate. American Journal of Medicine, 84, 951-954. 
Shiah, I.-S. \& Yatham, L. N. (2000) Serotonin in mania and in the mechanism of action of mood stabilizers: a review of clinical studies. Bipolar Disorder, 2, 77-92.

Suppes, T., Baldessarini, R. J., Faedda, G. L., et al (1991) Risk of recurrence following discontinuation of lithium treatment in bipolar disorder. Archives of General Psychiatry, 48, 10821088.

Thies-Flechtner, K., Muller-Oerlinghausen, B., Seibert, W., et al (1996) Effect of prophylactic treatment on suicide risk in patients with major affective disorders. Data from a randomized prospective trial. Pharmacopsychiatry, 29, 103-107.

Thompson, J. M., Gallagher, P., Hughes, J. H., et al (2005) Neurocognitive impairment in euthymic patients with bipolar affective disorder. British Journal of Psychiatry, 186, 32-40.

Tohen, M., Greil, W., Calabrese, J. R., et al (2005) Olanzapine versus lithium in the maintenance treatment of bipolar disorder: $\mathrm{a}$ 12-month randomized double-blind controlled clinical trial. American Journal of Psychiatry, 162, 1281-1290.

Tondo, L., Hennen, J., Baldessarini, R. J., et al (2001) Lower suicide risk with long-term lithium treatment in major affective illness: a meta-analysis. Acta Psychiatrica Scandinavica, 104, 163-172.

Wang, H., Lu, X. Q. \& Zhang, Y. F. (1997) Modulation of phosphatidylinositol turnover on central nicotinic receptors. Zhongruo Yao Li Xue Bao, 18, 341-344.

Wang, H. Y., Johnson, G. P. \& Friedman, E. (2001) Lithium treatment inhibits protein kinase $C$ translocation in rat brain cortex. Psychopharmacology, 159, 80-86.

Wood, A. J. \& Goodwin, G. M. (1987) A review of the biochemical and neuropharmacological actions of lithium. Psychological Medicine, 17, 579-600.

\section{MCQs}

1 The evidence base provides clear evidence that lithium is an efficacious treatment in the following situations:

a prophylactic treatment of bipolar disorder

b prophylactic treatment of unipolar disorder

c treatment of mania

d treatment of bipolar depression

e in monotherapy for schizophrenia.

2 The following are associated with severe lithium toxicity:

a cerebellar signs

b sinus and junctional bradycardia

c hypotension

d fine resting tremor

e acute renal failure.
3 Regarding the management of lithium toxicity:

a patients with suspected lithium toxicity should be observed for at least $24 \mathrm{~h}$

b a therapeutic level excludes toxicity

c serum lithium levels provide the only necessary guide to the severity of poisoning

d lithium excretion may be diminished in toxicity

e cerebellar symptoms may persist for months.

4 Regarding diabetes insipidus:

a it is characterised by polyuria and polydipsia

b it always results from renal tubular dysfunction

c it is usually neurogenic in origin when induced by lithium

$\mathrm{d}$ it may be diagnosed through the water deprivation test and the desmopressin (DDAVP) test

e polypharmacy including lithium is not a risk factor.

5 Regarding the lithium rebound phenomenon:

a abrupt discontinuation of lithium prophylaxis may precipitate early depressive recurrence in bipolar disorder

$\mathrm{b}$ the risk of depression is earlier and greater than the risk of mania

c Goodwin calculated that lithium prophylaxis for bipolar disorder must be maintained for at least 6 months before an overall advantage over no treatment is conferred

$\mathrm{d}$ the development of resistance to lithium treatment following discontinuation is an established clinical phenomenon

e gradual discontinuation of lithium has been demonstrated to lead to a higher rate of episode recurrence than abrupt withdrawal.

$\begin{array}{llllll}\text { MCQ answers } & & & \\ 1 & & 2 & 3 & 4 & 5 \\ \text { a T } & \text { a T } & \text { a T } & \text { a T } & \text { a T } \\ \text { b F } & \text { b T } & \text { b F } & \text { b F } & \text { b F } \\ \text { c T } & \text { c T } & \text { c F } & \text { c F } & \text { c F } \\ \text { d F } & \text { d F } & \text { d T } & \text { d T } & \text { d F } \\ \text { e F } & \text { e T } & \text { e T } & \text { e F } & \text { e F }\end{array}$

